\title{
Association between chloride-rich versus chloride-restrictive intravenous fluid administration and acute kidney injury in cardiovascular patients in ICU wards
}

\author{
XUDONG WANG, CHAO ZHANG, GUANGSU HUANG, DAHE HAN, XIAOYAN MENG, YI GUO and CHEN KAN \\ Department of Intensive Care Unit, The Second Affiliated Hospital of Xuzhou \\ Medical College, Xuzhou, Jiangsu 221006, P.R. China
}

Received January 11, 2016; Accepted June 6, 2016

DOI: $10.3892 /$ etm.2016.3431

\begin{abstract}
The aim of the study was to investigate the therapeutic effect of chloride-restrictive fluid to prevent acute kidney injury (AKI) in cardiovascular patients in intensive care unit (ICU) wards. Between January 2013 and September 2014, 456 patients admitted to ICU wards following diagnosis of cardiovascular disease were recruited and randomized to receive chloride-rich (232 patients) or chloride-restrictive (224 patients) fluid. The baseline characteristics and incidence of Kidney Disease Improving Global Outcomes (KDIGO)-defined AKI was then compared. No significant difference was identified in the baseline characteristics between the two groups. The incidence of moderate-to-severe KDIGO-defined AKI was significantly decreased in patients who received chloride-restrictive fluid. In conclusion, chloride-restrictive may be a novel effective intervention in preventing KDIGO-defined AKI in cardiovascular patients in ICU wards.
\end{abstract}

\section{Introduction}

The use of intravenous chloride is common in the clinical setting in intensive care unit (ICU) wards. However, chloride content has been reported to be associated with a number of unfavorable clinical outcomes. Previous findings have shown that chloride-rich fluid induced or exacerbated hyperchloremia and metabolic acidosis in critical patients, respectively $(1,2)$. The association between chloride and renal vasoconstriction and decreased glomerular filtration rate has been previously established (3-5). In patients who underwent major surgery, chloride fluid prolonged time to first micturition and decreased urine output $(6,7)$. Therefore, chloride-restrictive fluid has been

Correspondence to: Dr Xudong Wang, Department of Intensive Care Unit, The Second Affiliated Hospital of Xuzhou Medical College, 32 Meijian Road, Xuzhou, Jiangsu 221006, P.R. China E-mail: vnqlprin388@163.com

Key words: acute kidney injury, chloride-restrictive, cardiovascular, intensive care unit trialed to investigate the potential of replacing chloride-liberal fluid with chloride-restrictive fluid.

Results from a double-blind trial in 2012 showed that 2 liters of high-chloride fluid ( $0.9 \%$ saline) decreased cortical perfusion in human participants compared to a low-chloride fluid (Plasma-Lyte) (8). Similar results were obtained from another study which employed hydroxyethyl starch (HES) in a low chloride solution and compared the outcomes with the administration of HES in saline (9). Those results suggested the possible association between chloride content in intravenous fluid and decreased renal function, which may eventually lead to acute kidney injury (AKI). However, the clinical benefits of reducing chloride administration remain inconclusive. Previous studies that compared the clinical outcome of chloride-liberal and chloride-restrictive fluid administration in critically ill patients showed that the implementation of a chloride-restrictive strategy in a tertiary ICU was associated with a significant decrease in the incidence of AKI $(10,11)$.

In the present study, we performed a prospective randomized open-label study to investigate whether chloride-restrictive fluid was able to provide clinical benefits to critical cardiovascular patients in terms of reducing the incidence of AKI.

\section{Patients and methods}

Patient population and study design. Between January 2013 and September 2014, 456 patients diagnosed with with cardiovascular diseaes were admitted to ICU wards. The study was approved by the Human Research Ethics Committee of The Second Affiliated Hospital of Xuzhou Medical College (Jiangsu, China). Written consent was obtained from all the patients or legal guardians.

The patients in the control group (chloride-liberal group) received chloride-rich fluid as prescribed by physicians. The saline used included $0.9 \%$ saline (chloride concentration, $150 \mathrm{mmol} / \mathrm{l}$ ), $4 \%$ succinylated gelatin solution (chloride concentration, $120 \mathrm{mmol} / \mathrm{l}$ ), and $4 \%$ albumin in sodium chloride (chloride concentration, $128 \mathrm{mmol} / \mathrm{l}$ ). The patients in the intervention group (chloride-restrictive group) received low-chloride fluid as prescribed by physicians. The used fluids included a lactated crystalloid solution (chloride 
Table I. Baseline characteristics of the patients in chloride-rich and chloride-restrictive groups.

\begin{tabular}{lcr}
\hline Characteristics & Chloride-rich group (n=232) & Chloride-restrictive group (n=224) \\
\hline Male gender, no. $(\%)$ & $159(68.2 \%)$ & $348(66.4 \%)$ \\
Age, mean (range) & $62(26-75)$ & $67(23-76)$ \\
APACHE II score (range 0-71; mean, 95\% CI) & $15.7(15.4-16.2)$ & $16.2(15.6-16.8)$ \\
APACHE III score (range 0-300; mean, 95\% CI) & $58(56-61)$ & $58(56-60)$ \\
SAPS II (range 0-163; mean, 95\% CI) & $32(31-34)$ & $33(31-34)$ \\
Baseline creatinine level $(\mu$ mol/l) & $110(88-125)$ & $112(89-121)$ \\
Comorbidities & & $21(9.4 \%)$ \\
Severe sepsis or septic shock, $(\%)$ & $18(7.9 \%)$ & $6(2.7 \%)$ \\
Chronic lung disease, no. $(\%)$ & $7(3.0 \%)$ & $14(6.3 \%)$ \\
Chronic liver disease, no. $(\%)$ & $14(5.8 \%)$ & $9(4.0 \%)$ \\
Chronic renal failure, no. $(\%)$ & $8(3.6 \%)$ & $8(3.6 \%)$ \\
Immunosuppression, no. $(\%)$ & $8(3.6 \%)$ & $4(1.7 \%)$ \\
Metastatic cancer, no. $(\%)$ & $4(1.7 \%)$ & $1(0.4 \%)$ \\
Leukemia or myeloma, no. $(\%)$ & $1(0.4 \%)$ & \\
\hline
\end{tabular}

APACHE, Acute Physiology and Chronic Health Evaluation; SAPS, Simplified Acute Physiology Score; CI, confidence interval.

concentration, $109 \mathrm{mmol} / \mathrm{l})$, a balanced buffered solution (chloride concentration, $98 \mathrm{mmol} / \mathrm{l}$ ), and a $20 \%$ albumin solution (chloride concentration, $19 \mathrm{mmol} / \mathrm{l}$ ). The low-chloride fluid was exchanged with chloride-rich fluid only in the case of hyponatremia, traumatic brain injury, and cerebral edema under the prescription of specialists. The demographic information for all the patients enrolled was collected, including Acute Physiology and Chronic Health Evaluation (APACHE) II and III scores, Simplified Acute Physiology Score II (SAPS II), and multiple clinical characteristics of each admission. The renal function prior to admission to ICU was evaluated by examining serum creatinine levels. Serum creatinine levels were continually monitored during the patient's stay in the ICU ward. The incidence of acute kidney failure was defined as an increase in creatinine from baseline to ICU peak, injury, failure, loss, and end-stage system definition, as previously described $(12,13)$. The baseline creatinine level was defined as the lowest creatinine level prior to surgery or ICU stay.

Statistical analysis. Statistical analysis was performed using SPSS software 11.0 (SPSS, Inc., Chicago, IL, USA). Baseline comparisons were performed using the Chi-square test for equal proportion with results reported as numbers, percentages, and $95 \%$ confidence intervals. Continuously normally distributed variables were compared using t-tests and reported as means with $95 \%$ confidence intervals while non-normally distributed data were compared using the Wilcoxon rank sum test and reported as medians and interquartile range.

\section{Results}

Demographic information of patients. Between January 2013 and September 2014, 456 patients were admitted to ICU wards following diagnosis of cardiovascular disease. Of the
Table II. Incidence of acute kidney injury stratified by the KDIGO and serum creatinine criteria.

\begin{tabular}{lcc}
\hline Characteristics & $\begin{array}{c}\text { Chloride-rich } \\
\text { group }(\mathrm{n}=232)\end{array}$ & $\begin{array}{c}\text { Chloride-restrictive } \\
\text { group }(\mathrm{n}=224)\end{array}$ \\
\hline $\begin{array}{l}\text { KDIGO classfication } \\
\text { (no., \%) }\end{array}$ & \\
Stage 1 & $45(19.5 \%)$ & $35(15.6)$ \\
Stage 2 & $16(7.1 \%)$ & $12(5.3 \%)$ \\
Stage 3 & $38(16.4 \%)$ & $24(10.9 \%)$ \\
Stages 2 and 3 & $54(23.5 \%)$ & $36(18.1 \%)$ \\
RRT & $21(9.2 \%)$ & $12(5.5 \%)$ \\
\hline
\end{tabular}

KDIGO, Kidney Disease Improving Global Outcomes; RRT, renal replacement therapy.

456 patients with cardiovascular diagnosis, 232 patients received intravenous administration of chloride-rich fluid while the remaining 224 patients received chloride-restrictive fluid. The median follow-up time for patients in the control group was 12 months (9-15 months) and 11 months (9-15 months) for patients in teh intervention group. Demographic data of the patients are provided in Table I.

As shown in Table I, no significant difference was found with regard to characteristics including age, gender distribution, APACHE II score, SAPS II and baseline creatinine level between the chloride-rich and chloride-restrictive groups (all $\mathrm{P}>0.05$ ).

Subsequently, we analyzed the comorbidities of recruited patients and the results showed that the most common comorbidities were severe sepsis or septic shock, accounting for 7.9 and $9.4 \%$, respectively, of patients in the chloride-rich and chloride-restrictive groups, respectively. In addition, 
no significant difference was identified with regard to the incidence of the remaining comorbidities including, chronic lung disease, chronic liver disease, chronic renal failure, immunosuppression, metastatic cancer and leukemia or myeloma between the chloride-rich and chloride-restrictive groups. Thus, the demographic data show that patients in the two groups had comparable baseline characteristics.

Incidence of $A K I$. The incidence of AKI in the different groups is provdied in Table II. The degree of AKI was evaluated by Kidney Disease Improving Global Outcomes (KDIGO) and serum creatinine criteria. As shown in Table II, 45 patients in the chloride-rich group and 35 patients in the chloride-restrictive group had stage 1 kidney injury. Although fewer cases of stage 1 kidney injury were reported in patients receiving chloride-restrictive fluid, no significant difference was found in the incidence of stage 1 kidney injury between the two groups. Similar results were identified for the incidence of stage 2 kidney injury. Concerning stage 3 kidney injury, 38 cases were reported by patients who received chloride-rich fluid and 24 cases by patients who received chloride-restrictive fluid. The incidence of stage 3 kidney injury was significantly higher in patients receiving chloride-restrictive fluid $(\mathrm{P}<0.05)$. Additionally, chloride-restrictive fluid administration was associated with a significantly decreased incidence of moderate-to-severe (stages 2 and 3) KDIGO-defined AKI $(\mathrm{P}<0.05)$.

The use of renal replacement therapy (RRT) was also compared. However, no significant difference was identified in the RRT use between patients receiving chloride-rich and chloride-restrictive fluid.

Subsequently, we adjusted the incidence of AKI for gender, APACHE III score, and diagnosis and baseline creatinine level. The results showed that the risk of stages 2 and 3 of KDIGO-defined AKI was significantly reduced in patients receiving chloride-restrictive fluid $(\mathrm{P}<0.05)$.

\section{Discussion}

Diagnosis for ICU admission included, cardiovascular, gastrointestinal, metabolic, neurological, renal or genitourinary, and respiratory conditions. Of these conditions, cardiovascular condition was the most common reason for ICU admission. Given the close association between cardiovascular and renal function, we compared the therapeutic outcome of chloride-rich and chloride-restrictive fluid in ICU patients admitted due to cardiovascular conditions. Our results showed that chloride-restrictive fluid is effective in preventing AKI in ICU patients admitted due to cardiovascular causes.

AKI is a common complication for ICU with an incidence of $30-60 \%$ (14). Despite the advances in medical techniques, the mortality rate remains as high as $40-80 \%(14,15)$. Among patients admitted in the ICU, the ones who underwent elective surgery had a lower incidence of AKI, and the ones who had severe sepsis were associated with higher incidence. By contrast, the incidence of AKI induced by contrast agents was $11.5-19.0 \%$, which is lower than the overall incidence of AKI in ICU patients (16-20). The main pathogenesis of AKI in ICU patients included the decrease in the capability of excreting nitrogen-containing waste, adjusting electrolytes, balancing base and acid as well as body fluids. Use of effective intervention may prevent AKI, thus reducing the rate of mortality.

Earlier animal and human studies have shown that solutions with supraphysiological concentrations of chloride may induce detrimental renal effect (4-7). Yunos et al (1) also reported that a shorter time to micturition, improved urine output, and improved renal cortical perfusion, were associated with chloride-restrictive fluid, thus demonstrating its beneficial effect on renal function $(6,7)$. Furthermore, a large-scale study with 30,000 surgical patients showed that the chloride-rich fluid was significantly associated with increased risk of acute dialysis when compared with Plasma-Lyte administration (21).

In the present study, we recruited patients from ICU wards and randomly allocated the patients to receive chloride-rich and chloride-restrictive fluid. The results showed that the administration of chloride-restrictive fluid in ICU patients admitted for cardiovascular conditions significantly reduced the risk of moderate-to-severe (stages 2 and 3) KDIGO-defined AKI $(\mathrm{P}<0.05)$.

We also analyzed the incidence of AKI in different subgroups. First, the effect of age on the incidence of AKI was analyzed. It was identified that the incidence of AKI was significantly higher in patients $>50$ years of age in the two groups. The therapeutic benefit was also compared and the results showed that chloride-restrictive fluid was significantly more effective in reducing moderate-to-severe (stages 2 and 3) KDIGO-defined risk in younger patients $(<50$ years old) $(\mathrm{P}<0.05)$. By contrast, although chloride-restrictive fluid decreased the incidence of moderate-to-severe (stages 2 and 3 ) KDIGO-defined AKI in patients $>50$ years of age, the clinical outcome with regard to the incidence of moderate-to-severe (stages 2 and 3) KDIGO-defined AKI was not significantly different between the two groups $(\mathrm{P}>0.05)$. This result may be explained by the weak renal function of the older patients, who showed lower response to therapy.

The therapeutic effect of chloride-restrictive fluid was also different in male and female patients. No significant difference in the incidence of moderate-to-severe (stages 2 and 3) KDIGO-defined AKI between male and female patients was observed. However, the chloride-restrictive fluid was more effective to prevent moderate-to-severe (stages 2 and 3 ) KDIGO-defined AKI in female patients $(\mathrm{P}>0.05)$. In addition, the patients with one or more comorbidities were less sensitive to chloride-restrictive fluid compared to the patients with no comorbidities. At this stage it is difficult to explain the cause of different therapeutic effects in the various subgroups of patients. Further studies are required to investigate the optimal candidate for chloride-restrictive fluid therapy.

In summary, we conducted a controlled study on ICU patients admitted due to cardiovascular conditions and found that the implementation of chloride-restrictive fluid in patients is associated with a reduced risk of incidence of moderate-to-severe (stages 2 and 3) KDIGO-defined AKI compared to patients who received chloride-rich fluid.

\section{References}

1. Yunos NM, Kim IB, Bellomo R, Bailey M, Ho L, Story D, Gutteridge GA and Hart GK: The biochemical effects of restricting chloride-rich fluids in intensive care. Crit Care Med 39: 2419-2424, 2011. 
2. Smith RJ, Reid DA, Delaney EF and Santamaria JD: Fluid therapy using a balanced crystalloid solution and acid-base stability after cardiac surgery. Crit Care Resusc 12: 235-241, 2010.

3. Yunos NM, Bellomo R, Story D and Kellum J: Bench-to-bedside review: Chloride in critical illness. Crit Care 14: 226, 2010

4. Wilcox CS: Regulation of renal blood flow by plasma chloride. J Clin Invest 71: 726-735, 1983.

5. Bullivant EM, Wilcox CS and Welch WJ: Intrarenal vasoconstriction during hyperchloremia: Role of thromboxane. Am J Physiol 256: F152-F157, 1989.

6. Williams EL, Hildebrand KL, McCormick SA and Bedel MJ: The effect of intravenous lactated Ringer's solution versus $0.9 \%$ sodium chloride solution on serum osmolality in human volunteers. Anesth Analg 88: 999-1003, 1999.

7. Wilkes NJ, Woolf R, Mutch M, Mallett SV, Peachey T, Stephens R and Mythen MG: The effects of balanced versus saline-based hetastarch and crystalloid solutions on acid-base and electrolyte status and gastric mucosal perfusion in elderly surgical patients. Anesth Analg 93: 811-816, 2001

8. Chowdhury AH, Cox EF, Francis ST and Lobo DN: A randomized, controlled, double-blind crossover study on the effects of 2-L infusions of $0.9 \%$ saline and plasma-lyte ${ }^{\circledR} 148$ on renal blood flow velocity and renal cortical tissue perfusion in healthy volunteers. Ann Surg 256: 18-24, 2012.

9. Chowdhury AH, Cox EF, Francis ST and Lobo DN: A randomized, controlled, double-blind crossover study on the effects of 1-L infusions of $6 \%$ hydroxyethyl starch suspended in $0.9 \%$ saline (voluven) and a balanced solution (Plasma Volume Redibag) on blood volume, renal blood flow velocity, and renal cortical tissue perfusion in healthy volunteers. Ann Surg 259: 881-887, 2014

10. Yunos NM, Bellomo R, Glassford N, Sutcliffe H, Lam Q and Bailey M: Chloride-liberal vs. chloride-restrictive intravenous fluid administration and acute kidney injury: an extended analysis. Intensive Care Med 41: 257-264, 2015.

11. Yunos NM, Bellomo R, Hegarty C, Story D, Ho L and Bailey M Association between a chloride-liberal vs chloride-restrictive intravenous fluid administration strategy and kidney injury in critically ill adults. JAMA 308: 1566-1572, 2012.

12. Bellomo R, Ronco C, Kellum JA, Mehta RL and Palevsky P; Acute Dialysis Quality Initiative workgroup: Acute renal failure - definition, outcome measures, animal models, fluid therapy and information technology needs: the Second International Consensus Conference of the Acute Dialysis Quality Initiative (ADQI) Group. Crit Care 8: R204-R212, 2004.
13. Stevens PE and Levin A; Kidney Disease: Improving Global Outcomes Chronic Kidney Disease Guideline Development Work Group Members: Evaluation and management of chronic kidney disease: synopsis of the kidney disease: improving global outcomes 2012 clinical practice guideline. Ann Intern Med 158: $825-830,2013$

14. Singbartl K and Kellum JA: AKI in the ICU: Definition, epidemiology, risk stratification, and outcomes. Kidney Int 81: 819-825, 2012

15. Paudel MS, Wig N, Mahajan S, Pandey RM, Guleria R and Sharma SK: A study of incidence of AKI in critically ill patients. Ren Fail 34: 1217-1222, 2012.

16. Uchino S, Kellum JA, Bellomo R, Doig GS, Morimatsu H, Morgera S, Schetz M, Tan I, Bouman C, Macedo E, et al; Beginning and Ending Supportive Therapy for the Kidney (BEST Kidney) Investigators: Acute renal failure in critically ill patients: A multinational, multicenter study. JAMA 294: 813-818, 2005.

17. Odutayo A, Adhikari NK, Barton J, Burns KE, Friedrich JO, Klein D, Lapinsky S, Litwin S, Meret A, Moineddin R, et al: Epidemiology of acute kidney injury in Canadian critical care units: A prospective cohort study. Can J Anaesth 59: 934-942, 2012.

18. Negi S and Shigematsu T: Current therapeutic strategies for acute kidney injury. Clin Exp Nephrol 16: 672-678, 2012

19. Lin YF, Ko WJ, Chu TS, Chen YS, Wu VC, Chen YM, Wu MS, Chen YW, Tsai CW, Shiao CC, et al; NSARF Study Group: The 90-day mortality and the subsequent renal recovery in critically ill surgical patients requiring acute renal replacement therapy. Am J Surg 198: 325-332, 2009.

20. Tsagalis G: Update of acute kidney injury: Intensive care nephrology. Hippokratia 15 (Suppl 1): 53-68, 2011.

21. Shaw AD, Bagshaw SM, Goldstein SL, Scherer LA, Duan M, Schermer CR and Kellum JA: Major complications, mortality, and resource utilization after open abdominal surgery: $0.9 \%$ saline compared to Plasma-Lyte. Ann Surg 255: 821-829, 2012. 